\title{
Sharp blowup rate for NLS with a repulsive harmonic potential
}

Rui Zhou ${ }^{1 *}$

*Correspondence:

zhourui860514@163.com

'Department of Normal Education, Meishan Vocational and Technical

College, Meishan, 620010, P.R. China

\begin{abstract}
In this paper, we are concerned with the blowup solutions of the $L^{2}$ critical nonlinear Schrödinger equation with a repulsive harmonic potential. By using the results recently obtained by Merle and Raphaël and by Carles' transform we establish in a quite elementary way universal and sharp upper and lower bounds of the blowup rate for the blowup solutions of the aforementioned equation. As an application, we derive upper and lower bounds on the $L^{r}$-norms of the singular solutions.
\end{abstract}

MSC: $35 \mathrm{Q} 40 ; 35 \mathrm{Q} 41$

Keywords: Nonlinear Schrödinger equation; Repulsive harmonic potential; Singular solutions; Blowup rate

\section{Introduction}

In this paper, we study the following Cauchy problem:

$$
i \partial_{t} u+\frac{1}{2} \Delta u=-\frac{\omega^{2}}{2}|x|^{2} u-|u|^{4 / n} u, \quad x \in \mathbb{R}^{n}, t \in[0, T),
$$

where $\omega$ is a strictly positive parameter, $0<T \leq \infty, \Delta$ is the Laplace operator, and $u=$ $u(x, t)$ is a function of space and time. As a first motivation for studying (1.1), replacing the repulsive potential $-\frac{\omega^{2}}{2}|x|^{2}$ by $\frac{\omega^{2}}{2}|x|^{2}$, we get a model describing the Bose-Einstein condensate with attractive inter-particle interactions under a magnetic trap [14, 17].

We impose the initial data

$$
u_{\mid t=0}=u_{0}, \quad x \in \mathbb{R}^{n} .
$$

A variety of results exist in this case; we mention, for example, the local well-posedness for (1.1) established in [2]. Let $u_{0} \in \Sigma$. There exist $T>0$ and a unique maximal solution $u \in C([0, T] ; \Sigma)$ to the Cauchy problem (1.1). It is maximal in the sense that if $T<\infty$, then $u$ blows up at $T$, that is, $\lim _{t \rightarrow T}\left\|\nabla_{x} u(\cdot, t)\right\|_{L^{2}}=\infty$. Furthermore, for all $t \in[0, T)$ (the maximal existence interval), we have the following conservation laws.

(i) Conservation of mass:

$$
\|u(t)\|_{L^{2}}=\left\|u_{0}\right\|_{L^{2}} .
$$

(c) The Author(s) 2020. This article is licensed under a Creative Commons Attribution 4.0 International License, which permits use, sharing, adaptation, distribution and reproduction in any medium or format, as long as you give appropriate credit to the original author(s) and the source, provide a link to the Creative Commons licence, and indicate if changes were made. The images or other third party material in this article are included in the article's Creative Commons licence, unless indicated otherwise in a credit line to the material. If material is not included in the article's Creative Commons licence and your intended use is not permitted by statutory regulation or exceeds the permitted use, you will need to obtain permission directly from the copyright holder. To view a copy of this licence, visit http://creativecommons.org/licenses/by/4.0/. 
(ii) Conservation of energy:

$$
\begin{aligned}
H_{\omega}(u(t)) & =\frac{1}{2} \int\left|\nabla_{x} u\right|^{2} d x-\frac{\omega^{2}}{2} \int|x|^{2}|u|^{2} d x-\frac{1}{1+\frac{2}{n}} \int|u|^{2+\frac{4}{n}} d x \\
& =H_{\omega}\left(u_{0}\right) .
\end{aligned}
$$

The existence of blowup solutions for a class of initial data isproved in [1], and, last but not least, some dynamical properties of the blowup solutions were studied in $[6-8,18,20-22]$.

Another interesting aspect of this equation is its relation to the following problem known as the $L^{2}$-critical nonlinear Schrödinger equation:

$$
i \partial_{s} v+\frac{1}{2} \Delta v=-|v|^{4 / n} v, \quad y \in \mathbb{R}^{n}, s \in[0, S) .
$$

The link between problems (1.1) and (1.5) emanates from the work of Carles [2]. As for (1.1), the initial value problem (1.5) has been studied extensively; we refer the reader to [3] for the basic results. One of the major breakthrough as far was accomplished by Merle and Raphaël. In a series of papers [9-11], they succeeded in proving upper and lower bounds on the blowup rate suggested by numerical simulations for a class of initial data in $H^{1}\left(\mathbb{R}^{n}\right)$. This was done under some positivity condition on an explicit quadratic form. In this paper, we combine the ideas of Merle and Raphaël, Carles, and Zhu and Li to establish the exact upper and lower bounds on the blowup rate for the blowup solutions of (1.1).

The paper is organized as follows. In Sect. 2, we present some notations and the results obtained by Merle and Raphaël in connection with (1.5), and then we focus on Carles' work on the Cauchy problem (1.1). We conclude the section with the transform linking the two problems. In Sect. 3, we state the main results of this paper and proceed to their proofs. Section 4 is devoted to an application of our main theorems.

\section{Notations and preliminaries}

In this paper, for simplicity, we abbreviate $L^{r}\left(\mathbb{R}^{n}\right)$ and $H^{1}\left(\mathbb{R}^{n}\right)$ by $L^{r}$ and $H^{1}$, respectively. We define

(i) $\Sigma=\left\{u \in H^{1} ;|x| u \in L^{2}\right\}$ and

(ii) $(u, w)=\int_{\mathbb{R}^{n}} u(x) \bar{w}(x) d x, u, w \in L^{2}$.

We further recall some established facts about Cauchy problems (1.1) and (1.5) that are relevant in our study. First, we begin with Merle and Raphaël's celebrated result concerning the sharp upper and lower bounds on the blowup rate for blowup solutions to problem (1.5), and, second, we focus on Carles' work in relation with problem (1.1). We conclude the section with the relation between the two problems.

\subsection{Sharp blowup rate for Eq. (1.5)}

As mentioned in the introduction, the results of Merle and Raphaël were based on the following spectral property of some explicit quadratic form.

\subsubsection{Spectral property}

Let $n \geq 1$. Consider two real Schrödinger operators

$$
\mathcal{L}_{1}=-\Delta+\frac{2}{n}\left(\frac{4}{n}+1\right) Q^{\frac{4}{n}-1} y \cdot \nabla Q, \quad \mathcal{L}_{2}=-\Delta+\frac{2}{n} Q^{\frac{4}{n}-1} y \cdot \nabla Q,
$$


and the real quadratic form $H(\epsilon, \epsilon)=\left(\mathcal{L}_{1} \epsilon_{1}, \epsilon_{1}\right)+\left(\mathcal{L}_{2} \epsilon_{2}, \epsilon_{2}\right)$, where $\epsilon=\epsilon_{1}+i \epsilon_{2} \in H^{1}$. There exists a universal constant $\delta>0$ such that for all $\epsilon \in H^{1}$, if $\left(\epsilon_{1}, Q\right)=\left(\epsilon_{1}, Q_{1}\right)=\left(\epsilon_{1}, y Q\right)=$ $\left(\epsilon_{2}, Q_{1}\right)=\left(\epsilon_{2}, Q_{2}\right)=\left(\epsilon_{2}, \nabla Q\right)=0$, then

$$
H(\epsilon, \epsilon) \geq \delta\left(\int|\nabla \epsilon|^{2}+\int|\epsilon|^{2} e^{-2^{-}|y|} d y\right) .
$$

Here $Q$ is the ground state, that is, the unique $H^{1}$-positive and radially symmetric function solution to the scalar field equation $-\frac{1}{2} \Delta Q+Q-|Q|^{4 / n} Q=0$ (see $[5,15]$ for more detail), $Q_{1}=\frac{n}{2} Q+y \cdot \nabla Q, Q_{2}=\frac{n}{2} Q_{1}+y \cdot \nabla Q_{1}$, and $0<2^{-}<2$ is an arbitrary real number.

The above property was proved in [9] for $n=1 \mathrm{~m}$ wich is due to an explicit expression of the ground state in one dimension. In [4] the spectral property was checked numerically up to $n=5$.

Based on the spectral conjecture, Merle and Raphaël proved the following theorem.

Theorem 2.1 Let $n=1$ or $n \geq 2$ and assume that the spectral property holds. There exist $\alpha^{*}>0$ and a universal constant $C>0$ such that the following is true. Let $v(0, x)=u_{0} \in H^{1}$ be such that

$$
0<\alpha_{0}=\int\left|u_{0}\right|^{2} d x-\int Q^{2} d x<\alpha^{*}, \quad H_{0}\left(u_{0}\right)<\frac{1}{2}\left(\frac{\left|\operatorname{Im}\left(\int \overline{u_{0}} \nabla u_{0}\right)\right|}{\left\|u_{0}\right\|_{L^{2}}}\right)^{2} .
$$

Let $v$ be the unique maximal solution to Eq. (1.5) with initial data $v(0, x)=u_{0}$. Then $v$ blows up in finite time $S>0$, and

$$
\left\|\nabla_{y} v(s)\right\|_{L^{2}} \leq C\left(\frac{\ln |\ln (S-s)|}{S-s}\right)^{\frac{1}{2}} \quad \text { as } s \rightarrow S^{-}
$$

Theorem 2.1 states in particular that if the initial data $\psi \in H^{1}$ has a slightly supercritical mass and a strictly negative energy, then the corresponding maximal solution $v$ to Eq. (1.5) blows up in finite time with $\ln \ln$ rate of blowup. This is a step toward a proof of the negative energy conjecture. We note that the latter was proved in some particular cases by Ogawa and Tsutsumi $[12,13]$.

Let us now turn to the result of Merle and Raphaël [10] for the lower bound.

Theorem 2.2 Let $n=1$ or $n \geq 2$ and assume that the spectral property holds. There exist $\alpha^{*}>0$ and a universal constant $C>0$ such that the following is true. Let $v(0, x)=u_{0} \in H^{1}$ be such that

$$
0<\alpha_{0}=\int\left|u_{0}\right|^{2} d x-\int Q^{2} d x<\alpha^{*}
$$

Let $v$ be the unique maximal solution to Eq. (1.5) with initial data $v(0, x)=u_{0}$, and assume that it blows up in finite time $S>0$. Then we have the following lower bound on the blowup rate:

$$
\left\|\nabla_{y} v(s)\right\|_{L^{2}} \geq C\left(\frac{\ln |\ln (S-s)|}{S-s}\right)^{\frac{1}{2}} \quad \text { as } s \rightarrow S^{-} .
$$




\subsection{Carles' transform}

One of the main interests of problem (1.1) is its relation to (1.5). This was shown by Carles [2]. We have the following:

Proposition 2.3 Let $v$ solve the initial value problem (1.5). Define u by

$$
u(t, x)=\frac{1}{(\cosh (\omega t))^{n / 2}} e^{i \frac{\omega}{2}|x|^{2} \tanh (\omega t)} v\left(\frac{\tanh (\omega t)}{\omega}, \frac{x}{\cosh (\omega t)}\right) .
$$

Then $u$ solves (1.1). In particular, if the solution to (1.5) is unique, then so is the solution to (1.1), and it is given by (2.1).

Using transform (2.1), Carles proved the following:

Theorem 2.4 Let $u_{0} \in \Sigma$. Assume that the solution to problem (1.5) blows up at time $S>0$. Then:

(i) If $\omega \geq \frac{1}{S}$, then (1.1) has a unique global solution in $C\left(\mathbb{R}_{+}, \Sigma\right)$.

(ii) If $\omega<\frac{1}{S}$, then the solution to (1.1) blows up at time $\frac{\operatorname{argtanh}(\omega S)}{\omega}>S$.

\section{Sharp blow-up rate for Eq. (1.1)}

We come now to the principal section of this article. We begin with the following theorem on the sharp upper bound on the blow-up rate for singular solutions to (1.1).

Theorem 3.1 Let $n=1$ or $n \geq 2$ and assume that the spectral property holds. There exist $\alpha^{*}>0$ and a universal constant $C>0$ such that the following is true. Let $u_{0} \in \Sigma$ be such that

$$
0<\alpha_{0}=\int\left|u_{0}\right|^{2} d x-\int Q^{2} d x<\alpha^{*}, \quad H_{0}\left(u_{0}\right)<\frac{1}{2}\left(\frac{\left|\operatorname{Im}\left(\int \overline{u_{0}} \nabla u_{0}\right)\right|}{\left\|u_{0}\right\|_{L^{2}}}\right)^{2} .
$$

Then there exists $\omega_{0}>0$ such that for all $\left.\omega \in\right] 0, \omega_{0}[$, if $u$ is the unique maximal solution to (1.1) with initial data $u_{0}$, then $u$ blows up in finite time $T>0$, and

$$
\left\|\nabla_{x} u(t)\right\|_{L^{2}} \leq C\left(\frac{\ln |\ln (T-t)|}{T-t}\right)^{\frac{1}{2}} \text { as } t \rightarrow T^{-} .
$$

The proof of Theorem 3.1 is fairly easy; it is in fact a combination of the results presented in the previous section.

Proof Let $\alpha^{*}$ and $C$ be as in Theorem 2.1. Let $u_{0} \in \Sigma$ satisfy the above hypothesis. Let $v$ be the unique maximal solution to (1.5) with initial data $u_{0}$. We know from Theorem 2.1 that $v$ blows up in finite time $S>0$ and satisfies the following sharp upper bound on its blowup rate:

$$
\left\|\nabla_{y} v(s)\right\|_{L^{2}} \leq C\left(\frac{\ln |\ln (S-s)|}{S-s}\right)^{\frac{1}{2}} \text { as } s \rightarrow S^{-}
$$

Let $\omega_{0}>0$ be such that $\omega_{0} S<\frac{1}{2}$. Take $\left.\omega \in\right] 0, \omega_{0}$ [ and define $u$ by Carles' transform. It is clear that $u$ is the unique maximal solution to (1.1) with initial data $u_{0}$. Moreover, $u$ blows up in finite time $T=\frac{\arg \tanh (\omega S)}{\omega}$. 
We now proceed in two steps.

Step 1

Let $t \in[0, T)$. We have

$$
\begin{aligned}
\left\|\nabla_{y} v\left(\frac{\tanh (\omega t)}{\omega}\right)\right\|_{L^{2}} & \leq C\left(\frac{\ln \left|\ln \left(S-\frac{\tanh (\omega t)}{\omega}\right)\right|}{S-\frac{\tanh (\omega t)}{\omega}}\right)^{\frac{1}{2}} \\
& \leq C\left(\frac{\ln \left|\ln \left(\frac{\tanh (\omega T)-\tanh (\omega t)}{\omega}\right)\right|}{\frac{\tanh (\omega T)-\tanh (\omega t)}{\omega}}\right)^{\frac{1}{2}} \text { as } t \rightarrow T^{-} .
\end{aligned}
$$

A straightforward calculation gives

$$
\frac{\ln \left|\ln \left(\frac{\tanh (\omega T)-\tanh (\omega t)}{\omega}\right)\right|}{\frac{\tanh (\omega T)-\tanh (\omega t)}{\omega}} \underset{t \rightarrow T}{\sim} \frac{\ln \left|\ln \left(\frac{T-t}{\cosh ^{2}(\omega T)}\right)\right|}{\frac{T-t}{\cosh ^{2}(\omega T)}} .
$$

Hence

$$
\begin{aligned}
\left\|\nabla_{y} v\left(\frac{\tanh (\omega t)}{\omega}\right)\right\|_{L^{2}} & \leq C\left(\frac{\ln \left|\ln \left(\frac{T-t}{\cosh ^{2}(\omega T)}\right)\right|}{\frac{T-t}{\cosh ^{2}(\omega T)}}\right)^{\frac{1}{2}} \\
& \leq C \cosh (\omega T)\left(\frac{\ln \left|\ln \left(\frac{T-t}{\cosh ^{2}(\omega T)}\right)\right|}{T-t}\right)^{\frac{1}{2}}
\end{aligned}
$$

as $t \rightarrow T^{-}$. Note that

$$
\cosh (\omega T)=\cosh (\arg \tanh (\omega S)) \leq \cosh \left(\arg \tanh \left(\omega_{0} S\right)\right) \leq \cosh (\arg \tanh (1 / 2))
$$

so

$$
\left\|\nabla_{y} v\left(\frac{\tanh (\omega t)}{\omega}\right)\right\|_{L^{2}} \leq C\left(\frac{\ln \left|\ln \left(\frac{T-t}{\cosh ^{2}(\omega T)}\right)\right|}{T-t}\right)^{\frac{1}{2}} \text { as } t \rightarrow T^{-},
$$

with $C>0$ being a universal constant.

Elementary manipulations of the term $\ln \left|\ln \left(\frac{T-t}{\cosh ^{2}(\omega T)}\right)\right|$ show that it is less than $2 \ln |\ln (T-t)|$ for $t$ close enough (from the left) to $T$. Finally, we obtain the estimate

$$
\left\|\nabla_{y} v\left(\frac{\tanh (\omega t)}{\omega}\right)\right\|_{L^{2}} \leq C\left(\frac{\ln \mid \ln (T-t) \|}{T-t}\right)^{\frac{1}{2}} \text { as } t \rightarrow T^{-}
$$

for some universal constant $C$.

Step 2

Calculating $\nabla_{x} u$ using Carles' transform (2.1), we get

$$
\left\|\nabla_{x} u(t)\right\|_{L^{2}} \leq \omega \cosh (\omega t)\left\|y v\left(\frac{\tanh (\omega t)}{\omega}\right)\right\|_{L^{2}}+\frac{1}{\cosh (\omega t)}\left\|\nabla_{y} v\left(\frac{\tanh (\omega t)}{\omega}\right)\right\|_{L^{2}} .
$$

Using results from [16], we estimate the first term in the right hand-side of this inequality as follows:

$$
\left\|y v\left(\frac{\tanh (\omega t)}{\omega}\right)\right\|_{L^{2}}^{2} \leq C\left(\omega, u_{0}\right), \quad \forall t \in[0, T),
$$


where $C\left(\omega, u_{0}\right)$ denotes a constant depending on $\omega$ and $u_{0}$. Hence

$$
\left\|\nabla_{x} u(t)\right\|_{L^{2}} \leq \omega C\left(\omega, u_{0}\right) \cosh (\omega t)+\frac{1}{\cosh (\omega t)}\left\|\nabla_{y} v\left(\frac{\tanh (\omega t)}{\omega}\right)\right\|_{L^{2}}
$$

for all $t \in[0, T)$. We know from the previous step that $\cosh (\omega T) \leq \cosh (\arg \tanh (1 / 2))=M$, and hence for all $t \in[0, T)$,

$$
\left\|\nabla_{x} u(t)\right\|_{L^{2}} \leq \omega M C\left(\omega, u_{0}\right)+\frac{1}{\cosh (\omega t)}\left\|\nabla_{y} v\left(\frac{\tanh (\omega t)}{\omega}\right)\right\|_{L^{2}} .
$$

Since $\left\|\nabla_{x} u(t)\right\|_{L^{2}} \underset{t \rightarrow T}{\longrightarrow} \infty$, we have

$$
\left\|\nabla_{x} u(t)\right\|_{L^{2}} \leq \frac{1}{2}\left\|\nabla_{x} u(t)\right\|_{L^{2}}+\left\|\nabla_{y} v\left(\frac{\tanh (\omega t)}{\omega}\right)\right\|_{L^{2}}
$$

for $t$ close enough to $T$. Here we used the fact that $\cosh (x) \geq 1$ for all $x \in \mathbb{R}$. From (3.1) we arrive at

$$
\left\|\nabla_{x} u(t)\right\|_{L^{2}} \leq C\left(\frac{\ln \mid \ln (T-t) \|}{T-t}\right)^{\frac{1}{2}} \text { as } t \rightarrow T^{-},
$$

which is the announced bound. This completes the proof of Theorem 3.1

Now we state a result analogous to Theorem 2.2 for solutions to (1.1).

Theorem 3.2 Let $n=1$ or $n \geq 2$ and assume that the spectral property holds. Then there exist $\alpha^{*}>0$ and a universal constant $C>0$ such that the following is true. Let $u_{0} \in H^{1}$ be such that

$$
0<\alpha_{0}=\int\left|u_{0}\right|^{2} d x-\int Q^{2} d x<\alpha^{*} .
$$

Let $u$ be the unique maximal solution to (1.1) with initial data $u_{0}$, and assume that it blows up in finite time $T>0$. Then we have the following lower bound on the blowup rate:

$$
\left\|\nabla_{x} u(t)\right\|_{L^{2}} \geq C\left(\frac{\ln |\ln (T-t)|}{T-t}\right)^{\frac{1}{2}} \quad \text { as } t \rightarrow T^{-} .
$$

Proof Let $\alpha^{*}$ and $C$ be as in Theorem 2.2. Let $u_{0} \in \Sigma$ satisfy the above hypothesis. Let $u$ be the unique maximal solution to (1.1) with initial data $u_{0}$ and assume that it blows up in finite time $T$. Let $v$ be the unique maximal solution to (1.5) with initial data $u_{0}$ related to $u$ by Carles' transform (2.1). From Theorem 2.2, since $v$ blows up in finite time $S>0$, we deduce that it satisfies the following lower bound on its bolwup rate:

$$
\left\|\nabla_{y} v(s)\right\|_{L^{2}} \geq C\left(\frac{\ln |\ln (S-s)|}{S-s}\right)^{\frac{1}{2}} \text { as } s \rightarrow S^{-} .
$$

Here the blowup time is $S$ is given by Carles' transform

$$
S=\frac{\tanh (\omega T)}{\omega} .
$$


As in step 1 of the previous proof, after some elementary calculations, we get the estimate

$$
\left\|\nabla_{y} v\left(\frac{\tanh (\omega t)}{\omega}\right)\right\|_{L^{2}} \geq C\left(\frac{\ln \mid \ln (T-t) \|}{T-t}\right)^{\frac{1}{2}} \text { as } t \rightarrow T^{-}
$$

for some universal constant $C>0$. With the same notation as in the proof of Theorem 3.1, we get

$$
\begin{aligned}
\left\|\nabla_{x} u(t)\right\|_{L^{2}} & \geq \frac{1}{\cosh (\omega t)}\left\|\nabla_{y} v\left(\frac{\tanh (\omega t)}{\omega}\right)\right\|_{L^{2}}-\omega \cosh (\omega t)\left\|y v\left(\frac{\tanh (\omega t)}{\omega}\right)\right\|_{L^{2}} \\
& \geq \frac{1}{M}\left\|\nabla_{y} v\left(\frac{\tanh (\omega t)}{\omega}\right)\right\|_{L^{2}}-\omega M C\left(\omega, u_{0}\right) .
\end{aligned}
$$

Since $\left\|\nabla_{x} u(t)\right\|_{L^{2}} \underset{t \rightarrow T}{\longrightarrow} \infty$, we have

$$
\left\|\nabla_{x} u(t)\right\|_{L^{2}} \geq \frac{1}{M}\left\|\nabla_{y} v\left(\frac{\tanh (\omega t)}{\omega}\right)\right\|_{L^{2}}-\frac{1}{2}\left\|\nabla_{x} u(t)\right\|_{L^{2}} \quad \text { as } t \rightarrow T^{-} .
$$

Combining this inequality with (3.5), we obtain

$$
\left\|\nabla_{x} u(t)\right\|_{L^{2}} \geq C\left(\frac{\ln |\ln (T-t)|}{T-t}\right)^{\frac{1}{2}} \quad \text { as } t \rightarrow T^{-}
$$

for some universal constant $C>0$. This establishes the lower bound for singular solutions to $(1.1)$.

\section{Further discussion}

We simply obtain upper and lower bounds on the $L^{r}$-norms for blowup solutions without using the rate of $L^{2}$-concentration for NLS with potential established in [19].

Corollary 4.1 If all the assumptions in Theorem 3.1 are satisfied, then there exists a universal constant $C>0$ such that for any $r$ with $2<r<\infty$, we have

$$
\|u(t)\|_{L^{r}} \leq C\left(\frac{\ln |\ln (T-t)|}{T-t}\right)^{\frac{n(r-2)}{4 r}} \quad \text { as } t \rightarrow T^{-} .
$$

Proof We have the following Gagliardo-Nirenberg inequality

$$
\|u(t)\|_{L^{r}} \leq C\|u(t)\|_{L^{2}}^{1-\delta(r)}\left\|\nabla_{x} u(t)\right\|_{L^{2}}^{\delta(r)}, \quad \forall t \in[0, T)
$$

where $\delta(r)=n\left(\frac{1}{2}-\frac{1}{r}\right)$. Applying Theorem 3.1 yields

$$
\|u(t)\|_{L^{r}} \leq C\left\|u_{0}\right\|_{L^{2}}^{1-\delta(r)}\left(\frac{\ln |\ln (T-t)|}{T-t}\right)^{\frac{\delta(r)}{2}} \quad \text { as } t \rightarrow T^{-},
$$

where we used the conservation of mass.

A simple calculation gives $\frac{\delta(r)}{2}=\frac{n(r-2)}{4 r}$, which proves the desired result. 
Corollary 4.2 If all the assumptions in Theorem 3.2 are satisfied, then there exists a universal constant $C>0$ such that for any $r$ with $2<r<\infty$, we have

$$
\|u(t)\|_{L^{r}} \geq C\left(\frac{\ln |\ln (T-t)|}{T-t}\right)^{\frac{n(r-2)}{4 r}} \text { as } t \rightarrow T^{-} .
$$

Proof Suppose first that $2<r \leq 2+\frac{4}{n}$. This allows us to write the Gagliardo-Nirenberg inequality

$$
\|u(t)\|_{L^{2+\frac{4}{n}}}^{2+\frac{4}{n}} \leq C\left\|\nabla_{x} u(t)\right\|_{L^{2}}^{2-\mu}\|u(t)\|_{L^{r}}^{\frac{4}{n}+\mu}, \quad \forall t \in[0, T)
$$

where $\mu=\frac{4 r-8}{2 n-r(n-2)}$. By conservation of energy and the above inequality we obtain, for all $t \in[0, T)$,

$$
\begin{aligned}
\left\|\nabla_{x} u(t)\right\|_{L^{2}}^{2} \leq & 2 H_{\omega}\left(u_{0}\right)+\omega^{2}\|x u(t)\|_{L^{2}}^{2}+\frac{2}{1+\frac{2}{n}}\|u(t)\|_{L^{2+\frac{4}{n}}}^{2+\frac{4}{n}} \\
\leq & 2 H_{0}\left(u_{0}\right)+\omega^{2} \cosh ^{2}(\omega t)\left\|y v\left(\frac{\tanh (\omega t)}{\omega}\right)\right\|_{L^{2}}^{2} \\
& +\frac{2 C}{1+\frac{2}{n}}\left\|\nabla_{x} u(t)\right\|_{L^{2}}^{2-\mu}\|u(t)\|_{L^{r}}^{\frac{4}{n}+\mu},
\end{aligned}
$$

where in the second inequality, we used the relation

$$
\|x u(t)\|_{L^{2}}^{2}=\cosh ^{2}(\omega t)\left\|y v\left(\frac{\tanh (\omega t)}{\omega}\right)\right\|_{L^{2}}^{2}
$$

and the fact that $H_{\omega}\left(u_{0}\right) \leq H_{0}\left(u_{0}\right)$. Using the same notations as in the previous section, we have, for all $t \in[0, T)$,

$$
\left\|y v\left(\frac{\tanh (\omega t)}{\omega}\right)\right\|_{L^{2}}^{2} \leq C\left(\omega, u_{0}\right)
$$

and $\cosh (\omega t) \leq \cosh (\arg \tanh (1 / 2))=M$. Therefore

$$
\left\|\nabla_{x} u(t)\right\|_{L^{2}}^{2} \leq 2 H_{0}\left(u_{0}\right)+M^{2} \omega^{2} C\left(\omega, u_{0}\right)+\frac{2 C}{1+\frac{2}{n}}\left\|\nabla_{x} u(t)\right\|_{L^{2}}^{2-\mu}\|u(t)\|_{L^{r}}^{\frac{4}{n}+\mu}
$$

for all $t \in[0, T)$. Since $\left\|\nabla_{x} u(t)\right\|_{L^{2}} \underset{t \rightarrow T}{\longrightarrow} \infty$, we have

$$
\left\|\nabla_{x} u(t)\right\|_{L^{2}}^{2} \leq \frac{1}{2}\left\|\nabla_{x} u(t)\right\|_{L^{2}}^{2}+\frac{2 C}{1+\frac{2}{n}}\left\|\nabla_{x} u(t)\right\|_{L^{2}}^{2-\mu}\|u(t)\|_{L^{r}}^{\frac{4}{n}+\mu} \quad \text { as } t \rightarrow T^{-}
$$

or, equivalently,

$$
\left\|\nabla_{x} u(t)\right\|_{L^{2}} \leq C\|u(t)\|_{L^{r}}^{\frac{4}{n^{r}+\mu}} \quad \text { as } t \rightarrow T^{-}
$$


for some universal constant $C>0$. Since $\frac{\frac{4}{n}+\mu}{\mu} \delta(r)=1$, we get

$$
\left\|\nabla_{x} u(t)\right\|_{L^{2}}^{\delta(r)} \leq C\|u(t)\|_{L^{r}} \quad \text { as } t \rightarrow T^{-}
$$

We conclude using the lower bound on the blowup rate. Suppose now $2+\frac{4}{n}<r<\infty$. A similar argument as before, combined with the following Hölders inequality, yields the result:

$$
\|u(t)\|_{L^{2+\frac{4}{n}}}^{2+\frac{4}{n}} \leq C\|u(t)\|_{L^{r}}^{\frac{4 r}{n(r-2)}}\|u(t)\|_{L^{2}}^{\frac{2\left(r-\left(\frac{4}{n}+2\right)\right)}{r-2}}, \quad \forall t \in[0, T) .
$$

\section{Acknowledgements}

The author would like to thank Dr. B. Abdelwahab for his encouragement and important references.

\section{Funding}

This work was supported by Sichuan Sciences and Technology Program (granted No. 2020YJ0146)

\section{Availability of data and materials}

Not applicable.

\section{Competing interests}

The author declares that she has no competing interests.

\section{Authors' contributions}

The author read and approved the final manuscript.

\section{Publisher's Note}

Springer Nature remains neutral with regard to jurisdictional claims in published maps and institutional affiliations.

Received: 29 May 2020 Accepted: 10 December 2020 Published online: 04 January 2021

\section{References}

1. Carles, R:: Critical nonlinear Schrödinger equations with and without harmonic potential. Math. Models Methods Appl. Sci. 12(10), 1513-1523 (2002)

2. Carles, R.: Nonlinear Schrödinger equations with repulsive harmonic potential and applications. SIAM J. Math. Anal. 35(4), 823-843 (2003)

3. Cazenave, T.: Semilinear Schrödinger Equations. Courant Lecture Notes in Mathematics, vol. 10. New York University Courant Institute of Mathematical Sciences, New York (2003)

4. Fibich, G., Merle, F., Raphaël, P.: Proof of a spectral property related to the singularity formation for the $L^{2} \mathrm{critical}$ nonlinear Schrödinger equation. Physica D 200(1), 1-13 (2006)

5. Kwong, M.K.: Uniqueness of positive solutions of $\Delta u-u+u^{p}=0$ in $\mathbb{R}^{n}$. Arch. Ration. Mech. Anal. 105(3), 243-266 (1989)

6. Leng, L., Li, X.G., Zheng, P.: Sharp criteria for the nonlinear Schrödinger equation with combined nonlinearities of power-type and Hartree-type. Appl. Anal. 96(16), 2846-2851 (2017)

7. Li, X.G., Zhang, J.: Limit behavior of blow-up solutions for critical nonlinear Schrödinger equation with harmonic potential. Differ. Integral Equ. 19(7), 761-771 (2006)

8. Li, X.G., Zhang, J., Chen, G.G.: L²-concentration of blow-up solutions for the nonlinear Schrödinger equations with harmonic potential. Chin. J. Contemp. Math. 26, 31-38 (2005)

9. Merle, F., Raphaeël, P.: Blow-up dynamic and upper bound on the blow-up rate for critical nonlinear Schrödinger equation. Ann. Math. 1, 157-222 (2005)

10. Merle, F., Raphaeël, P.: On a sharp lower bound on the blow-up rate for the $L^{2}$-critical nonlinear Schrödinger equation. J. Am. Math. Soc. 19, 37-90 (2006)

11. Merle, F., Raphaël, P.: Sharp upper bound on the blow up rate for critical nonlinear Schrödinger equation. Geom Funct. Anal. 13(3), 591-642 (2003)

12. Ogawa, T., Tsutsumi, Y.: Blow-up of $H^{1}$ solutions for the one-dimensional nonlinear Schrödinger equations with critical power nonlinearity. Proc. Am. Math. Soc. 111(2), 487-496 (1991)

13. Ogawa, T., Tsutsumi, Y.: Blow-up of $H^{1}$ solution for the nonlinear Schrödinger equation. J. Differ. Equ. 92(2), 317-330 (1991)

14. Oh, Y.G.: Cauchy problem and Ehrenfest law of nonlinear Schrödinger equations with potentials. J. Differ. Equ. 81(2), 255-274 (1989)

15. Strauss, W.A.: Existence of solitary waves in higher dimensions. Commun. Math. Phys. 55(2), 149-162 (1977)

16. Weinstein, M.I.: Nonlinear Schrödinger equations and sharp interpolation estimates. Commun. Math. Phys. 87 567-576 (1983)

17. Zhang, J.: Stability of attractive Bose-Einstein condensate. J. Stat. Phys. 101(3), 731-746 (2000)

18. Zhang, J.: Sharp threshold for blow-up and global existence in nonlinear Schrödinger equations under a harmonic potential. Commun. Partial Differ. Equ. 30, 1429-1443 (2005) 
19. Zhang, J., Zhu, S., Li, X.G.: Rate of $L^{2}$-concentration of the blow-up solution for critical nonlinear Schrödinger equation with potential. Math. Control Relat. Fields 1(1), 19-127 (2011)

20. Zhu, S.H.: On the blow-up solutions for the nonlinear fractional Schrödinger equation. J. Differ. Equ. 261(2), 1506-1531 (2016)

21. Zhu, S.H.: Existence of stable standing waves for the fractional Schrödinger equations with combined nonlinearities. J. Evol. Equ. 17(3), 1003-1021 (2017)

22. Zhu, S.H., Zhang, J., Li, X.G.: Limiting profile of blow-up solutions for the Gross-Pitaevskii equation. Sci. China Ser. A, Math. 52(5), 1017-1030 (2009)

Submit your manuscript to a SpringerOpen ${ }^{\circ}$ journal and benefit from:

- Convenient online submission

- Rigorous peer review

- Open access: articles freely available online

- High visibility within the field

- Retaining the copyright to your article

Submit your next manuscript at $\boldsymbol{\Delta}$ springeropen.com 